\title{
Stellar weak decay rates in neutron-deficient medium-mass nuclei
}

\author{
P. Sarriguren \\ Instituto de Estructura de la Materia, Consejo Superior de Investigaciones Científicas, Serrano 123, E-28006 Madrid, Spain
}

(Received 5 November 2010; revised manuscript received 5 January 2011; published 2 February 2011)

\begin{abstract}
Weak decay rates under stellar density and temperature conditions holding at the rapid proton-capture process are studied in neutron-deficient medium-mass waiting-point nuclei extending from Ni up to Sn. Neighboring isotopes to these waiting-point nuclei are also included in the analysis. The nuclear structure part of the problem is described within a deformed Skyrme Hartree-Fock + BCS + quasiparticle random-phase-approximation approach, which reproduces not only the $\beta$-decay half-lives but also the available Gamow-Teller strength distributions, measured under terrestrial conditions. The various sensitivities of the decay rates to both density and temperature are discussed. In particular, we study the impact of contributions coming from thermally populated excited states in the parent nucleus and the competition between $\beta$ decays and continuum electron captures.
\end{abstract}

DOI: 10.1103/PhysRevC.83.025801

PACS number(s): 23.40.-s, 21.60.Jz, 26.30.Ca, 27.50.+e

\section{INTRODUCTION}

An accurate understanding of most astrophysical processes necessarily requires information from nuclear physics, which provides the input to deal with network calculations and astrophysical simulations (see Refs. [1,2] and references therein). Obviously, nuclear physics uncertainties will finally affect the reliability of the description of those astrophysical processes. This is especially relevant in the case of explosive phenomena, which involves knowledge of the properties of exotic nuclei, which are not well explored yet. Thus, most of the astrophysical simulations of these violent events must be built on nuclearmodel predictions of limited quality and accuracy. This is particularly the case of the X-ray bursts (XRBs) [3-6], which are generated by a thermonuclear runaway in the hydrogenrich environment of an accreting neutron star that is fed from a red giant binary companion close enough to allow for mass transfer.

Type I XRBs are typically characterized by a rapid increase in luminosity generating burst energies of $10^{39}-10^{40}$ ergs, which are typically a factor 100 larger than the steady luminosity. The luminosity suffers a sharp rise of about 1-10 s followed by a gradual softening with time scales between 10 and $100 \mathrm{~s}$. These bursts are recurrent with time scales ranging from hours to days. The properties of XRBs are particularly dependent on the accretion rate. Typical accretion rates for type I XRBs are about $10^{-8}-10^{-9} M_{\odot} \mathrm{yr}^{-1}$. Lower accretion rates lead to weaker flashes, while larger accretion rates lead to stable burning on the surface of the neutron star.

The ignition of XRBs takes place when the temperature $T$ and the density $\rho$ in the accreted disk become high enough to allow a breakout from the hot CNO cycle. Peak conditions of $T=1-3 \mathrm{GK}$ and $\rho=10^{6}-10^{7} \mathrm{~g} \mathrm{~cm}^{-3}$ are reached, and eventually, this scenario allows the development of the nucleosynthesis rapid proton-capture $(r p)$ process [5-8], which is characterized by proton-capture reaction rates that are orders of magnitude faster than any other competing process, in particular $\beta$ decay. It produces rapid nucleosynthesis on the proton-rich side of stability toward heavier proton-rich nuclei, reaching nuclei with $A \gtrsim 100$, as shown in Ref. [9], where the $r p$ process ends in a closed SnSbTe cycle. It also explains the energy and luminosity profiles observed in XRBs.

Nuclear reaction network calculations, which may involve as much as several thousand nuclear processes, are performed to follow the time evolution of the isotopic abundances, to determine the amount of energy released by nuclear reactions, and to find the reaction path for the $r p$ process [3-10]. In general, the reaction path follows a series of fast proton-capture reactions until the drip line is reached and further proton capture is inhibited by a strong reverse photodisintegration reaction. At this point, the process may only proceed through a $\beta$ decay or a less probable double proton capture. Then the reaction flow has to wait for a relatively slow $\beta$ decay, and the respective nucleus is called a waiting point (WP). The short time scale of the $r p$ process (around $100 \mathrm{~s}$ ) makes highly significant any mechanism that may affect the process for several seconds, and the half-lives of the WP nuclei are of this order. Therefore, the half-lives of the WP nuclei along the reaction path determine the time scale of the nucleosynthesis process and the produced isotopic abundances. In this respect, the weak decay rates of neutron-deficient medium-mass nuclei under stellar conditions play a relevant role in understanding the $r p$ process.

Although the products of the nucleosynthesis $r p$ process are not expected to be ejected from type I XRBs due to the strength of the neutron star gravitational field, there are other speculative sites for the occurrence of $r p$ processes. This is the case of core collapse supernovae, which might supply suitable physical conditions for the $r p$ process provided neutrino-induced reactions are included in the nucleosynthesis calculations [11]. These reactions have to be included to bypass the slow $\beta$ decays at the WP nuclei via capture reactions of neutrons, which are created from the antielectron neutrino absorption by free protons [12]. Contrary to the XRBs, these scenarios will finally lead to the ejection of the nucleosynthetic products and thus contribute to the galactic chemical evolution.

Since the pioneering work of Fuller, Fowler, and Newman [10], where the general formalism to calculate 
weak-interaction rates in stellar environments as a function of density and temperature was introduced, improvements have been focused on the description of the nuclear structure aspect of the problem. Different approaches to describe the nuclear structure involved in the stellar weak decay rates can be found in the literature. They are basically divided into shell model $[13,14]$ and quasiparticle random-phase-approximation (QRPA) [15-17] categories. Certainly, the nuclear structure problem involved in the calculation of these rates must be treated in a reliable way. In particular, this implies that the nuclear models should be able to describe at least the experimental information available on the decay properties (Gamow-Teller strength distributions and $\beta$-decay half-lives) measured under terrestrial conditions. Although these decay properties may be different at the high $\rho$ and $T$ existing in $r p$ process scenarios, success in describing the decay properties in terrestrial conditions is a requirement for a reliable calculation of the weak decay rates in more general conditions. With this aim in mind, we study here the dependence of the decay rates on both $\rho$ and $T$ using a QRPA approach based on a self-consistent deformed Hartree-Fock (HF) mean field.

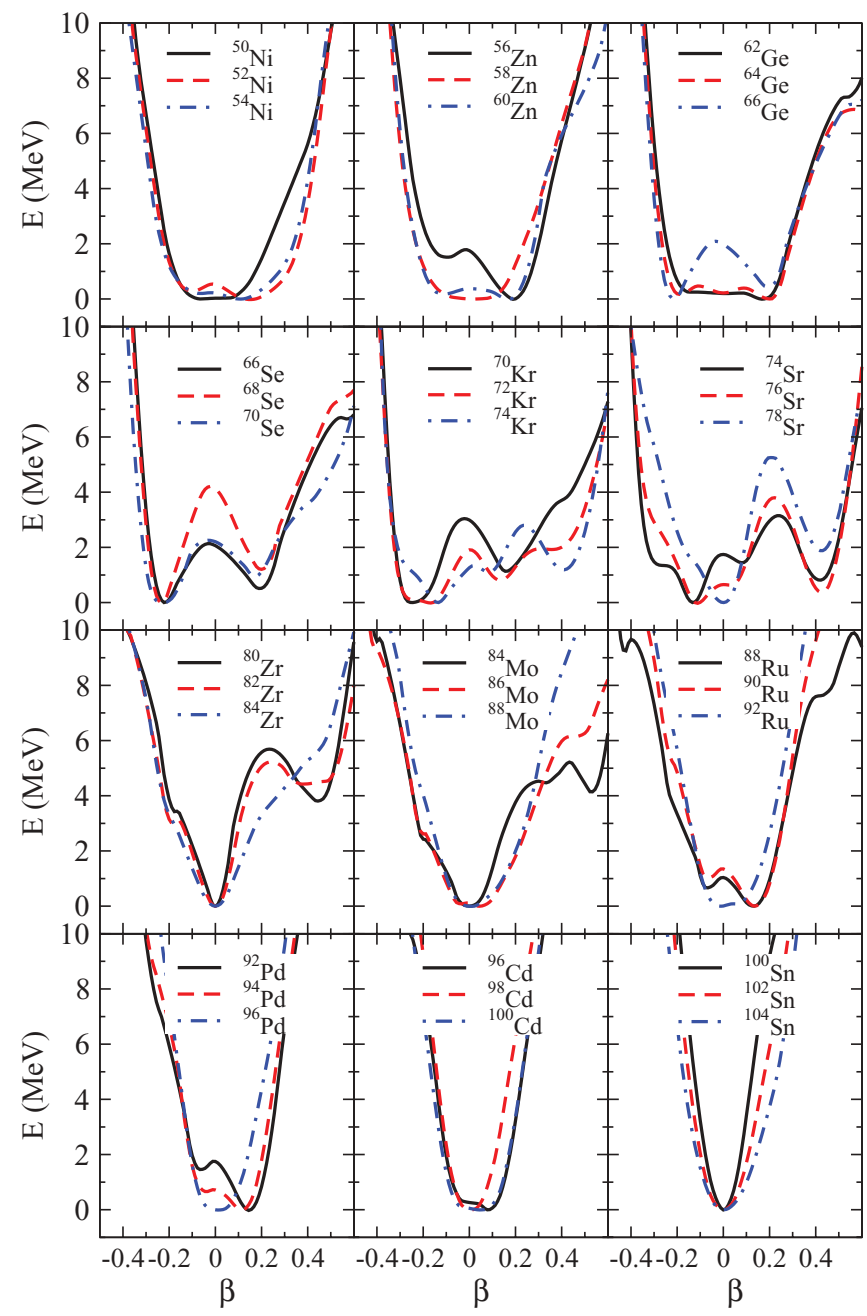

FIG. 1. (Color online) Potential energy curves for the even-even isotopes considered in this work obtained from constrained HF + BCS calculations with the Skyrme force SLy4.
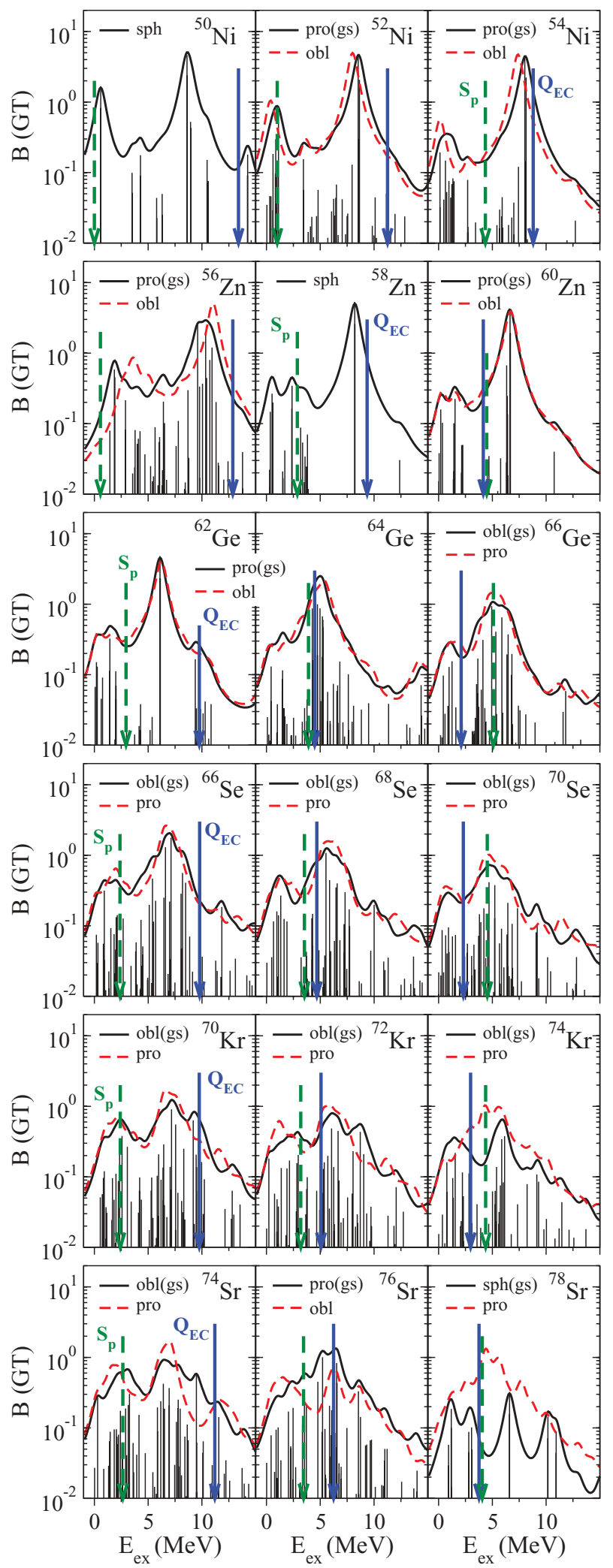

FIG. 2. (Color online) Calculated GT strength distributions for $\mathrm{Ni}, \mathrm{Zn}, \mathrm{Ge}, \mathrm{Se}, \mathrm{Kr}$, and $\mathrm{Sr}$ isotopes obtained from their ground states and from the shape-coexisting states. The individual strengths correspond to the ground states, whereas folded distributions are shown for the various configurations considered in each isotope. $Q_{\mathrm{EC}}$ values and proton separation energies $S_{p}$ are shown by vertical lines. 

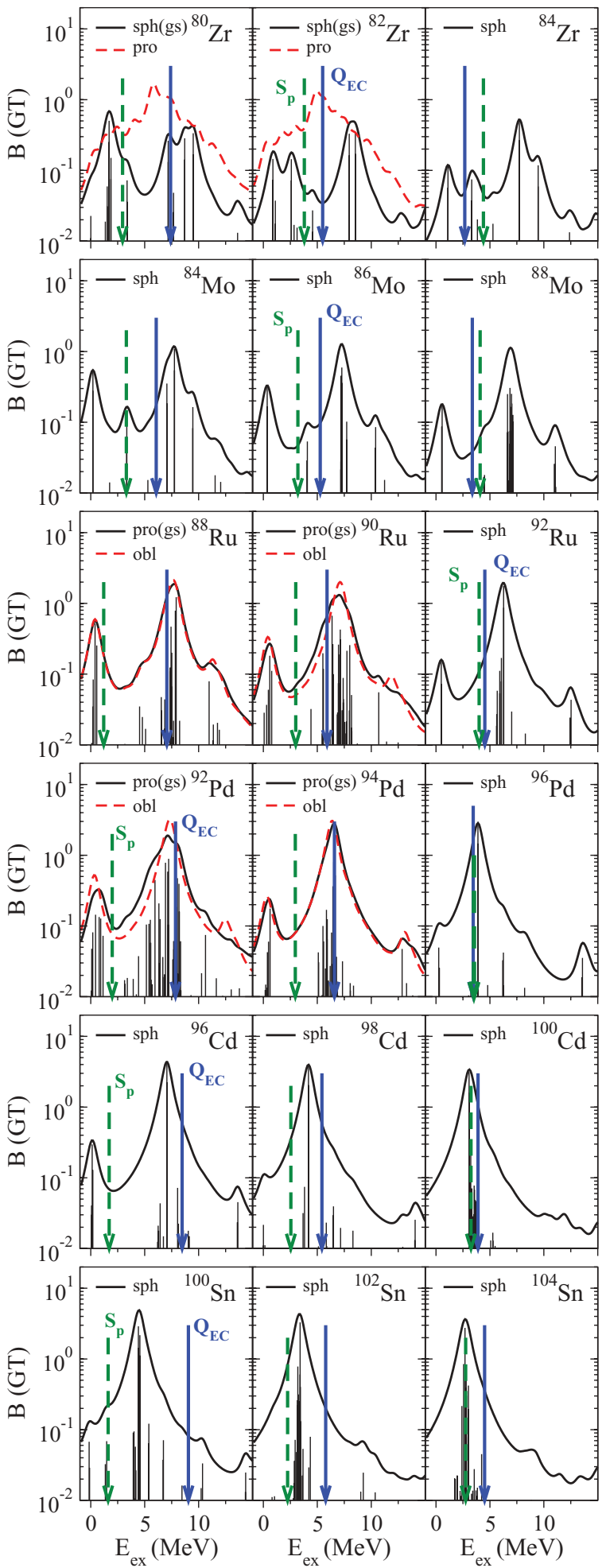

FIG. 3. (Color online) Same as in Fig. 2 but for $\mathrm{Zr}, \mathrm{Mo}, \mathrm{Ru}, \mathrm{Pd}$, $\mathrm{Cd}$, and $\mathrm{Sn}$ isotopes.

Deformation has to be taken into account because the reaction path in the $r p$ process crosses a region of highly deformed nuclei around $A=70-80$. This nuclear model has been tested successfully (see Ref. [18] and references therein) and reproduces very reasonably the experimental information

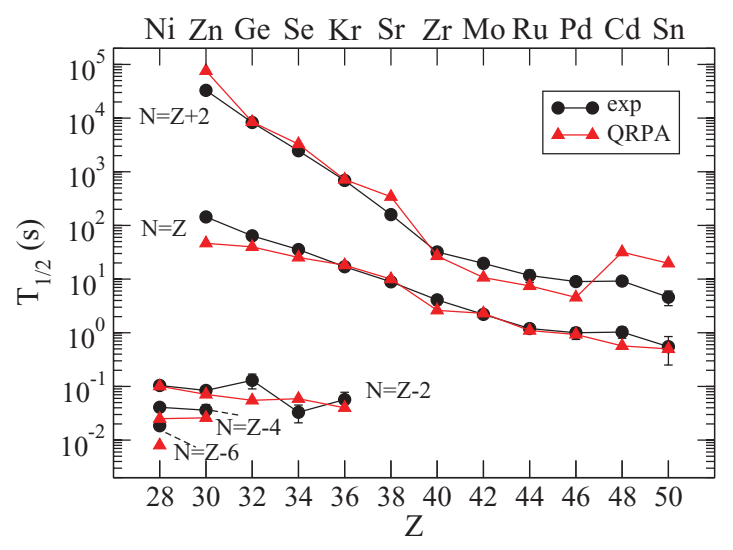

FIG. 4. (Color online) Calculated QRPA half-lives compared to experimental values.

available on both bulk and decay properties of medium-mass nuclei. In this work we focus our attention on the even-even WP Ni, Zn, Ge, Se, Kr, Sr, Zr, Mo, Ru, Pd, Cd, and Sn isotopes and their closest even-even neighbors.

This paper is organized as follows. In Sec. II the weak decay rates are introduced as functions of density and temperature, and their nuclear structure and phase-space components are studied. Section III contains the results. We study the decay properties first under terrestrial conditions and second as functions of both densities and temperatures of the $r p$ process. Section IV contains the conclusions of this work.

\section{WEAK DECAY RATES}

There are several distinctions between terrestrial and stellar decay rates caused by the effects of high $\rho$ and $T$. The main effect of $T$ is directly related to the thermal population of excited states in the decaying nucleus, accompanied by the corresponding depopulation of the ground states. The weak decay rates of excited states can be significantly different from those of the ground state, and a case-by-case consideration is needed. Another effect related to the high $\rho$ and $T$ comes from the fact that atoms in these scenarios are completely ionized, and consequently, electrons are no longer bound to the nuclei but form a degenerate plasma obeying a Fermi-Dirac distribution. This opens the possibility for continuum electron capture (CEC), in contrast to the orbital electron capture (OEC) produced by bound electrons in an atom under terrestrial conditions. These effects make weak interaction rates in the stellar interior sensitive functions of $T$ and $\rho$, with $T=1.5 \mathrm{GK}$ and $\rho=10^{6} \mathrm{~g} \mathrm{~cm}^{-3}$ as the most significant conditions for the $r p$ process [5].

The decay rate of the parent nucleus is given by

$$
\lambda=\sum_{i} \lambda_{i} \frac{2 J_{i}+1}{G} e^{-E_{i} /(k T)},
$$

where $G=\sum_{i}\left(2 J_{i}+1\right) e^{-E_{i} /(k T)}$ is the partition function, $J_{i}\left(E_{i}\right)$ is the angular momentum (excitation energy) of the parent nucleus state $i$, and thermal equilibrium is assumed. In principle, the sum extends over all populated states in the 

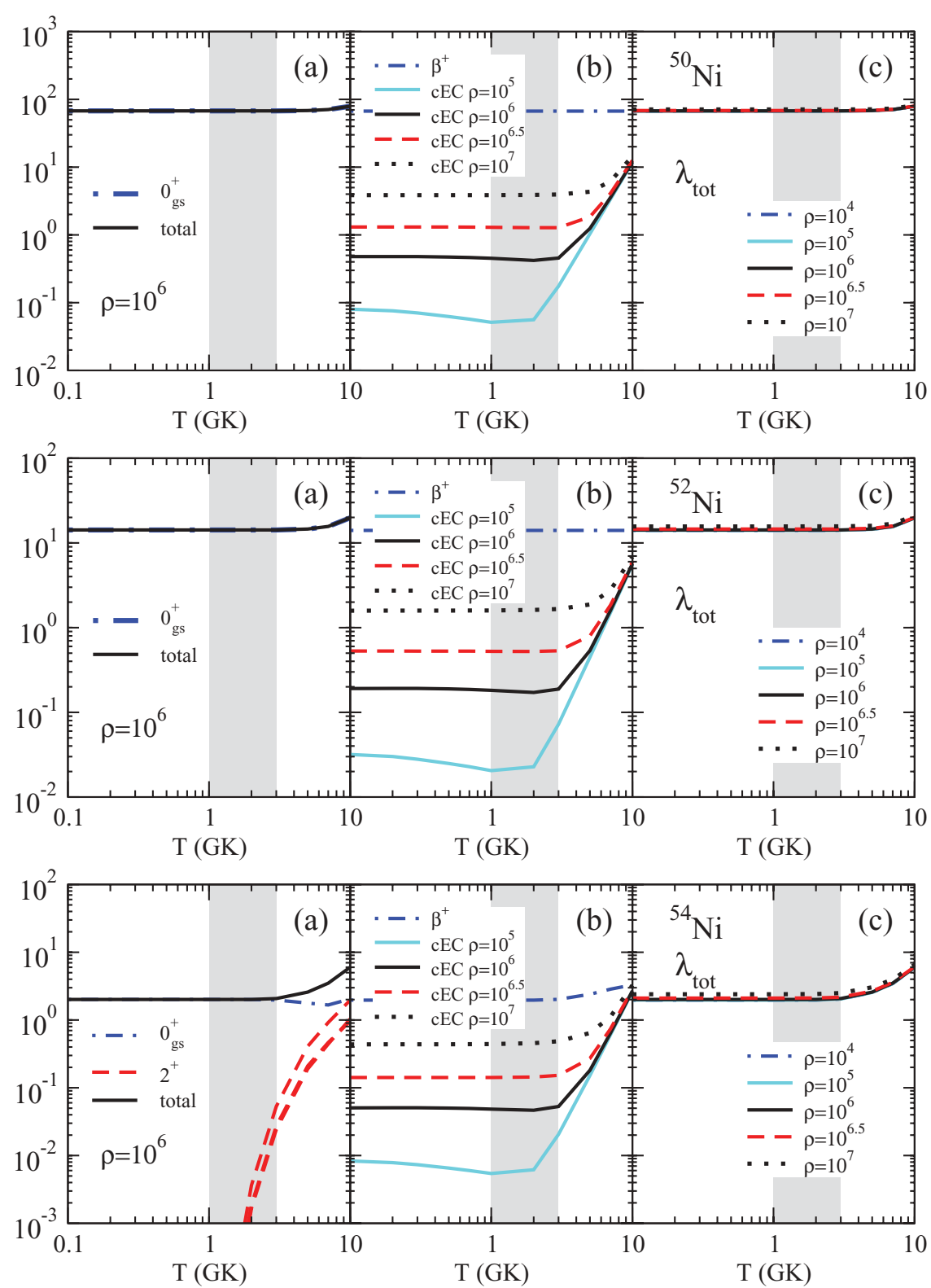

FIG. 5. (Color online) Decay rates $\left(\mathrm{s}^{-1}\right)$ of ${ }^{50,52,54} \mathrm{Ni}$ isotopes as a function of the temperature $T$ (GK). (a) Decomposition of the total rates into their contributions from the decays of the ground and excited $2^{+}$states. (b) Decomposition of the rates into their $\beta^{+}$ and CEC components evaluated at different densities. (c) Total rates at various densities. The label $\rho$ stands for $\rho Y_{e}\left(\mathrm{~mol} / \mathrm{cm}^{3}\right)$ (see text). parent nucleus up to the proton separation energy. However, since the range of temperatures for the $r p$ process peaks at $T=1.5 \mathrm{GK}(k T \sim 300 \mathrm{keV})$, only a few low-lying excited states are expected to contribute significantly to the decay. Specifically, we consider in this work all the collective lowlying excited states below $1 \mathrm{MeV}$ [19]. Two-quasiparticle excitations in even-even nuclei will appear at an excitation energy above $2 \mathrm{MeV}$, which is a typical energy to break a Cooper pair in these isotopes. Hence, they can be safely neglected at these temperatures. For example, the maximum population appears for the lowest of these states $\left(E_{2^{+}}=\right.$ $261 \mathrm{keV}$ in ${ }^{76} \mathrm{Sr}$ ), which at $T=1.5 \mathrm{GK}$ is $12 \%$, while the ground state still contributes $88 \%$.

The decay rate for the parent state $i$ is given by

$$
\lambda_{i}=\sum_{f} \lambda_{i f},
$$

where the sum extends over all the states in the final nucleus reached in the decay process. The rate $\lambda_{i f}$ from the initial state $i$ to the final state $f$ is given by

$$
\lambda_{i f}=\frac{\ln 2}{D} B_{i f} \Phi_{i f}(\rho, T),
$$

where $D=6146 \mathrm{~s}$. This expression is decomposed into a nuclear structure part $B_{i f}$, which contains the transition probabilities for allowed Fermi (F) and Gamow-Teller (GT) transitions,

$$
B_{i f}=B_{i f}(\mathrm{GT})+B_{i f}(\mathrm{~F})
$$

and a phase-space factor $\Phi_{i f}$, which is a sensitive function of $\rho$ and $T$. The theoretical description of both $B_{i f}$ and $\Phi_{i f}$ are explained next. 

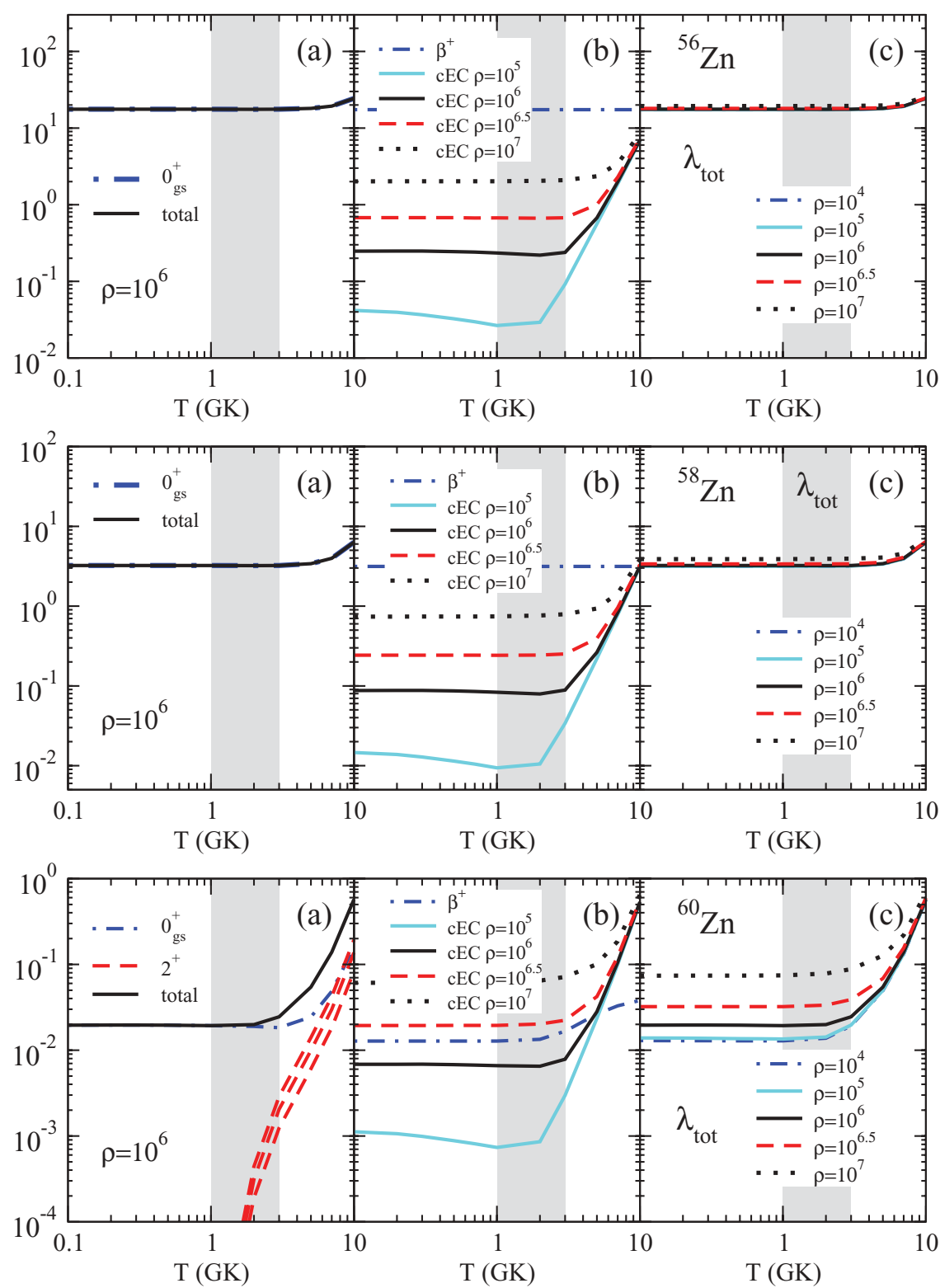

FIG. 6. (Color online) Same as in Fig. 5 but for ${ }^{56,58,60} \mathrm{Zn}$ isotopes.

\section{A. Nuclear structure}

The nuclear structure part of the problem is described within the QRPA formalism. Various approaches have been developed in the past to describe the spin-isospin nuclear excitations in QRPA [20-31]. In this subsection we show briefly the theoretical framework used in this paper to describe the nuclear part of the decay rates in the neutron-deficient nuclei considered in this work. More details of the formalism can be found in Refs. [29-31].

The method starts with a self-consistent deformed HartreeFock mean-field formalism obtained with Skyrme interactions, including pairing correlations. The single-particle energies, wave functions, and occupation probabilities are generated from this mean field. In this work we have chosen the Skyrme force SLy4 [32] as a representative of the Skyrme forces. This particular force includes some selected properties of unstable nuclei in the adjusting procedure of the parameters. It is one of the most successful Skyrme forces and has been extensively studied in recent years.

The solution of the HF equation is found by using the formalism developed in Ref. [33], assuming time reversal and axial symmetry. The single-particle wave functions are expanded in terms of the eigenstates of an axially symmetric harmonic oscillator in cylindrical coordinates, using 12 major shells. The method also includes pairing between like nucleons in BCS approximation with fixed gap parameters for protons and neutrons, which are determined phenomenologically from the odd-even mass differences involving the experimental binding energies [34].

The potential energy curves are analyzed as a function of the quadrupole deformation $\beta$,

$$
\beta=\sqrt{\frac{\pi}{5}} \frac{Q_{0}}{A\left\langle r^{2}\right\rangle},
$$



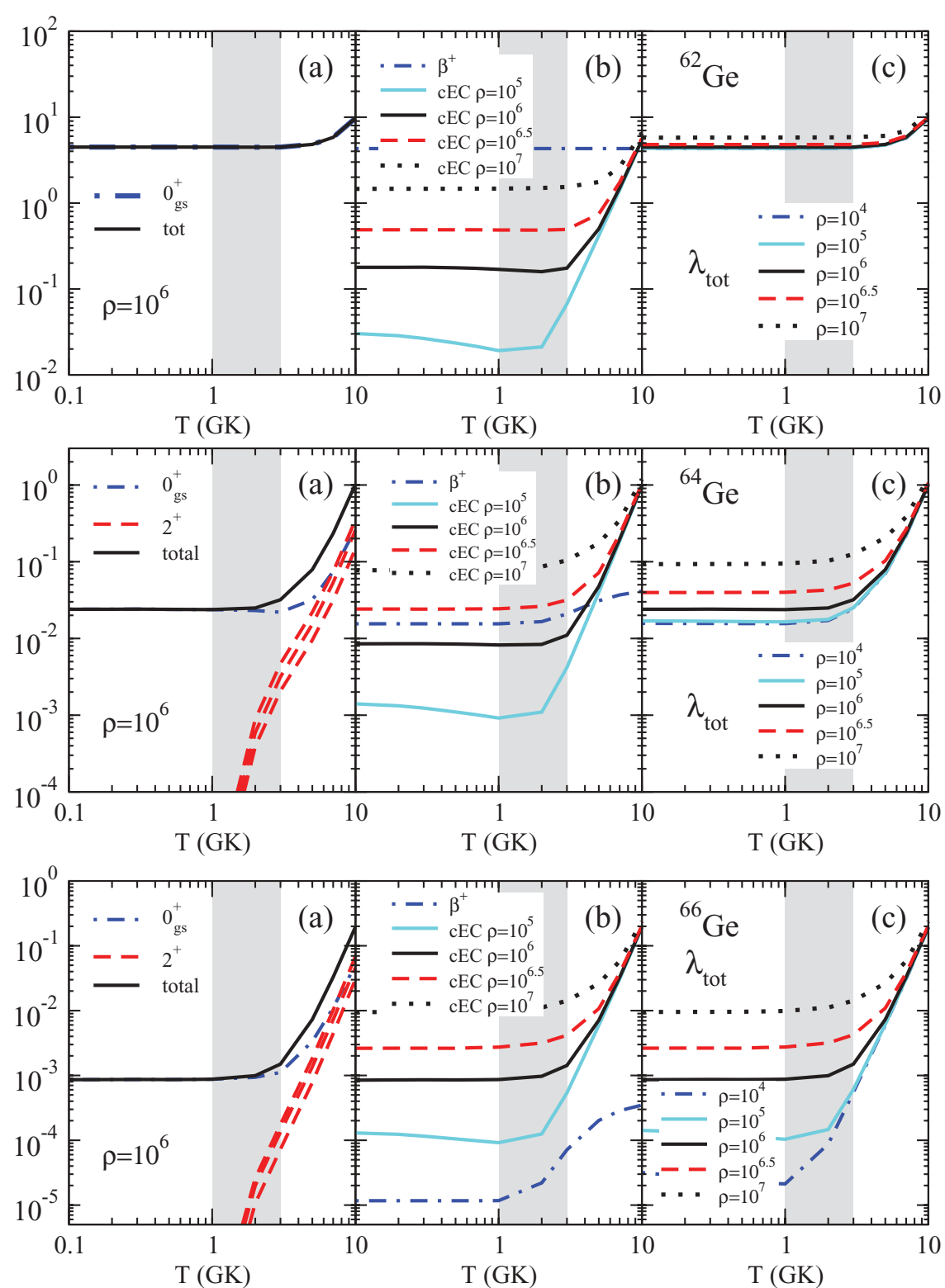

FIG. 7. (Color online) Same as in Fig. 5 but for ${ }^{62,64,66} \mathrm{Ge}$ isotopes. written in terms of the mass quadrupole moment $Q_{0}$ and the mean square radius $\left\langle r^{2}\right\rangle$. For that purpose, constrained HF calculations are performed with a quadratic constraint [35]. The HF energy is minimized under the constraint of keeping fixed the nuclear deformation. Calculations for GT strengths are performed subsequently for the various equilibrium shapes of each nucleus, that is, for the solutions, which are generally deformed, for which minima are obtained in the energy curves. Since decays connecting different shapes are disfavored, similar shapes are assumed for the ground state of the parent nucleus and for all populated states in the daughter nucleus. The validity of this assumption was discussed, for example, in Refs. [20,24].

To describe GT transitions, a spin-isospin residual interaction is added to the mean field and treated in a deformed proton-neutron QRPA. This interaction contains two parts, a particle-hole (ph) part and a particle-particle (pp) part. The interaction in the ph channel is responsible for the position and structure of the GT resonance [24,36], and it can be derived consistently from the same Skyrme interaction used to generate the mean field, through the second derivatives of the energy density functional with respect to the one-body densities. The ph residual interaction is finally expressed in a separable form by averaging the resulting contact force over the nuclear volume [29]. The pp part is a neutron-proton pairing force in the $J^{\pi}=1^{+}$coupling channel, which is also introduced as a separable force $[23,30]$. The strength of the pp residual interaction in this theoretical approach is not derived self-consistently from the SLy4 force used to obtain the mean field, but nevertheless, it has been fixed in accordance to it. This strength is usually fitted to reproduce globally the experimental half-lives. Various attempts have been made in the past to fix this strength [24], arriving at expressions that depend on the model used to describe the mean field, which is the Nilsson model in Ref. [24]. In previous works [30,36-39] we have studied the sensitivity of the GT strength distributions to the 

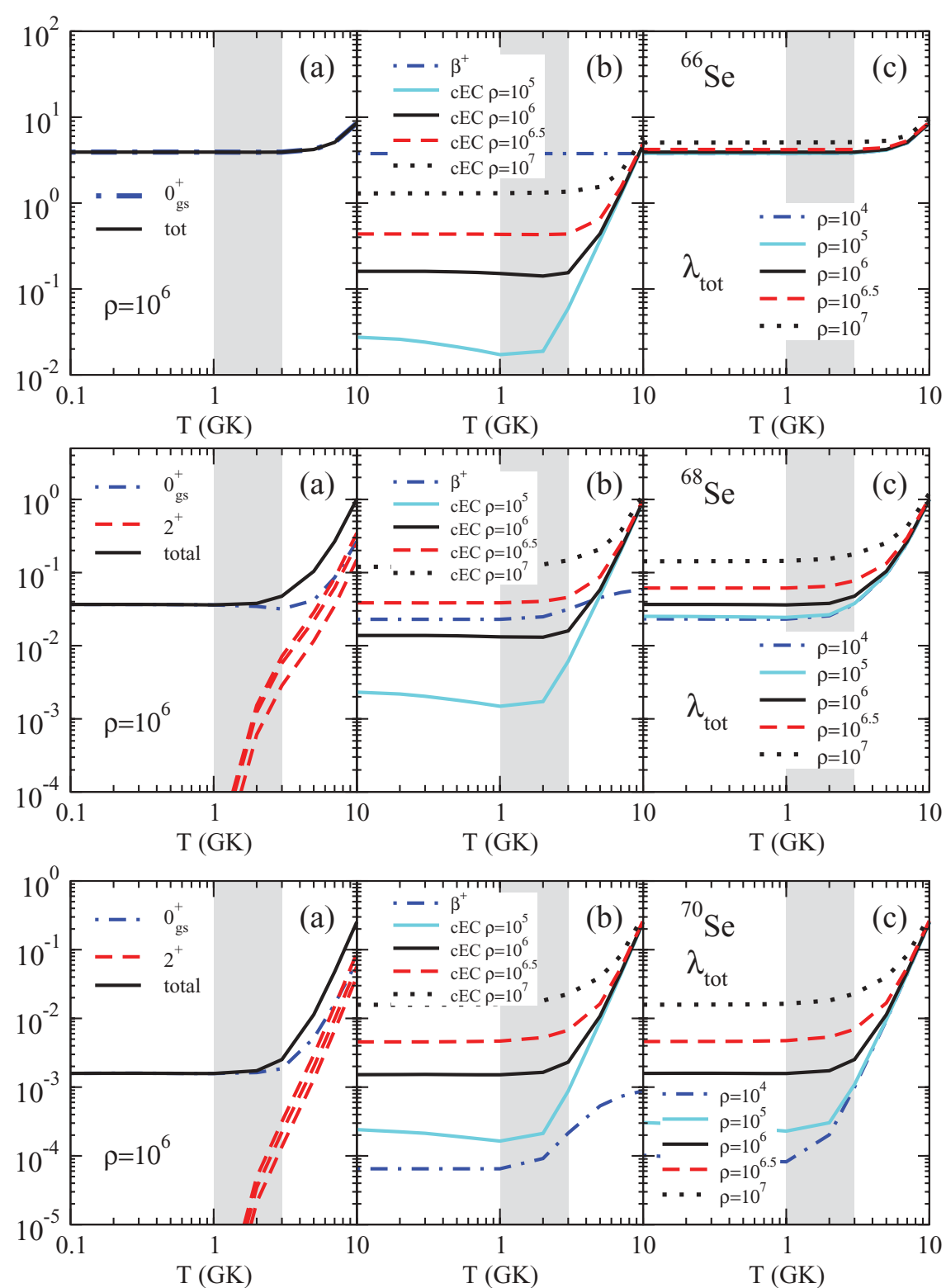

FIG. 8. (Color online) Same as in Fig. 5 but for ${ }^{66,68,70} \mathrm{Se}$ isotopes. various ingredients contributing to the deformed QRPA-like calculations, namely, to the nucleon-nucleon effective force, to pairing correlations, and to residual interactions. We found different sensitivities to them. In this work, all of these ingredients have been fixed to the most reasonable choices found previously [18] and mentioned here. In particular, we use the coupling strengths $\chi_{\mathrm{GT}}^{\mathrm{ph}}=0.15 \mathrm{MeV}$ and $\kappa_{\mathrm{GT}}^{\mathrm{pp}}=$ $0.03 \mathrm{MeV}$.

The proton-neutron QRPA phonon operator for GT excitations in even-even nuclei is written as

$$
\Gamma_{\omega_{K}}^{+}=\sum_{\pi \nu}\left[X_{\pi \nu}^{\omega_{K}} \alpha_{\nu}^{+} \alpha_{\bar{\pi}}^{+}+Y_{\pi \nu}^{\omega_{K}} \alpha_{\bar{\nu}} \alpha_{\pi}\right]
$$

where $\alpha^{+}(\alpha)$ are quasiparticle creation (annihilation) operators, $\omega_{K}$ are the QRPA excitation energies, and $X_{\pi \nu}^{\omega K}$ and $Y_{\pi \nu}^{\omega K}$ are the forward and backward amplitudes, respectively. For even-even nuclei the allowed GT transition amplitudes in the intrinsic frame connecting the QRPA ground state
$|0\rangle\left(\Gamma_{\omega K}|0\rangle=0\right)$ to one-phonon states $|\omega K\rangle\left(\Gamma_{\omega K}^{+}|0\rangle=|\omega K\rangle\right)$ are given by

$$
\left\langle\omega_{K}\left|\sigma_{K} t^{ \pm}\right| 0\right\rangle=\mp M_{ \pm}^{\omega_{K}}, \quad K=0,1
$$

where

$$
\begin{aligned}
& M_{-}^{\omega_{K}}=\sum_{\pi v}\left(q_{\pi v} X_{\pi v}^{\omega_{K}}+\tilde{q}_{\pi \nu} Y_{\pi v}^{\omega_{K}}\right), \\
& M_{+}^{\omega_{K}}=\sum_{\pi v}\left(\tilde{q}_{\pi v} X_{\pi \nu}^{\omega_{K}}+q_{\pi \nu} Y_{\pi v}^{\omega_{K}}\right),
\end{aligned}
$$

with

$$
\tilde{q}_{\pi v}=u_{\nu} v_{\pi} \Sigma_{K}^{v \pi}, \quad q_{\pi v}=v_{\nu} u_{\pi} \Sigma_{K}^{v \pi},
$$

$v$ being occupation amplitudes $\left(u^{2}=1-v^{2}\right)$, and $\Sigma_{K}^{v \pi}$ being spin matrix elements connecting neutron and proton states with spin operators

$$
\Sigma_{K}^{v \pi}=\left\langle v\left|\sigma_{K}\right| \pi\right\rangle
$$



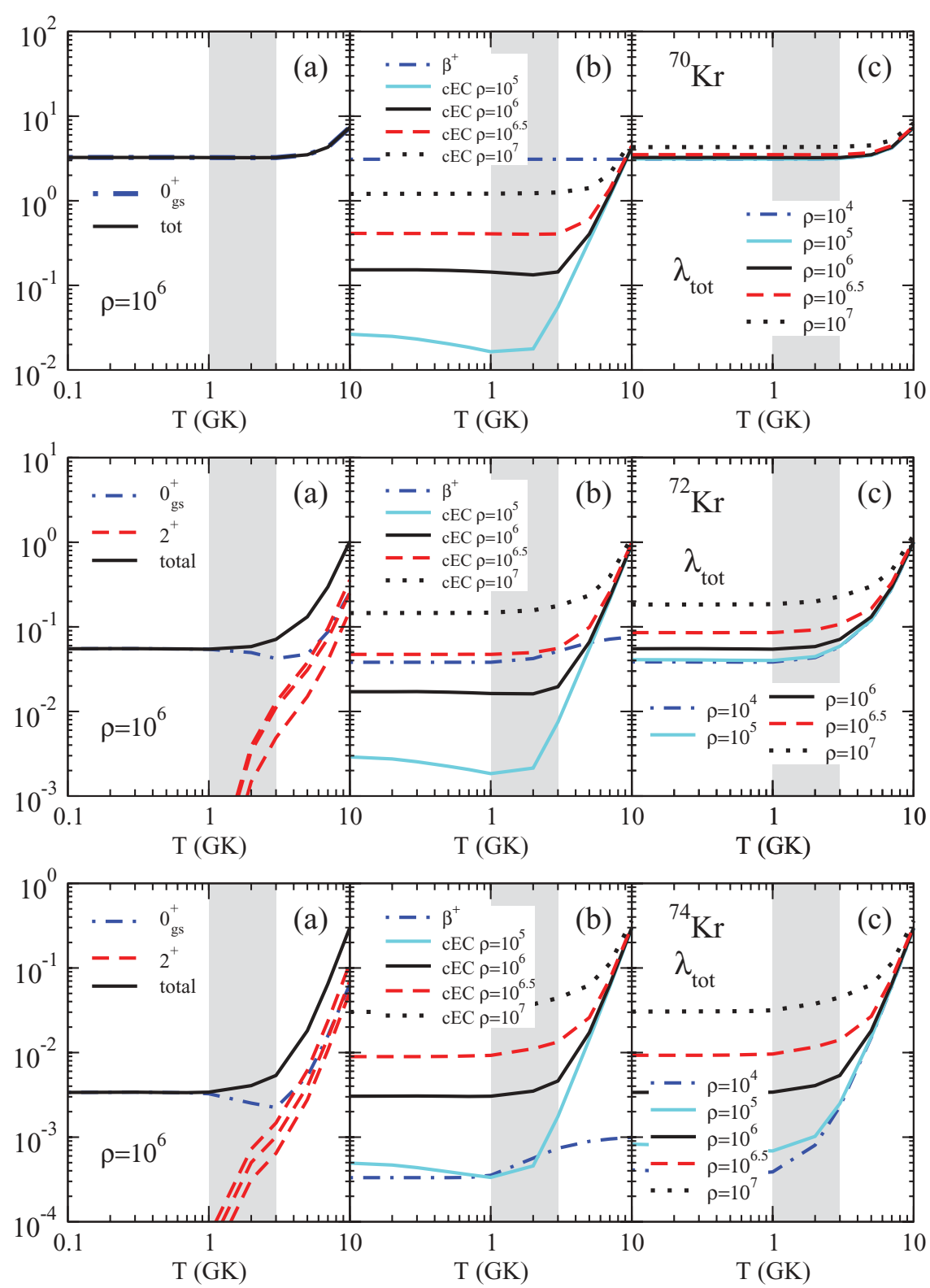

FIG. 9. (Color online) Same as in Fig. 5 but for ${ }^{70,72,74} \mathrm{Kr}$ isotopes.
The GT strength for a transition from an initial state $i$ to a final state $f$ is given by

$$
B_{i f}\left(\mathrm{GT}^{ \pm}\right)=\frac{1}{2 J_{i}+1}\left(\frac{g_{A}}{g_{V}}\right)_{\mathrm{eff}}^{2}\left\langle f\left\|\sum_{j}^{A} \sigma_{j} t_{j}^{ \pm}\right\| i\right\rangle^{2}
$$

where $\left(g_{A} / g_{V}\right)_{\text {eff }}=0.74\left(g_{A} / g_{V}\right)_{\text {bare }}$ is an effective quenched value. For the transition $I_{i} K_{i}\left(0^{+} 0\right) \rightarrow I_{f} K_{f}\left(1^{+} K\right)$ in the laboratory system, the energy distribution of the GT strength $B_{\omega}\left(\mathrm{GT}^{ \pm}\right)$is expressed in terms of the intrinsic amplitudes in Eq. (7) as

$$
\begin{aligned}
B_{\omega}\left(\mathrm{GT}^{ \pm}\right)= & \left(\frac{g_{A}}{g_{V}}\right)_{\text {eff }}^{2} \sum_{\omega_{K}}\left[\left\langle\omega_{K}\left|\sigma_{0} t^{ \pm}\right| 0\right\rangle^{2} \delta_{K, 0}\right. \\
& \left.+2\left\langle\omega_{K}\left|\sigma_{1} t^{ \pm}\right| 0\right\rangle^{2} \delta_{K, 1}\right] .
\end{aligned}
$$

To obtain this expression, the initial and final states in the laboratory frame have been expressed in terms of the intrinsic states using the Bohr-Mottelson factorization [40].
Concerning Fermi transitions, the Fermi operator is the isospin ladder operator $T_{ \pm}$, which commutes with the nuclear part of the Hamiltonian excluding the small Coulomb component. Then, superallowed Fermi transitions $\left(0^{+} \rightarrow 0^{+}\right)$ only occur between members of an isospin multiplet. The Fermi strength is narrowly concentrated in the isobaric analog state (IAS) of the ground state of the decaying nucleus. Thus, neglecting effects from isospin mixing, one has

$$
B_{i f}\left(F^{ \pm}\right)=\frac{1}{2 J_{i}+1}\left\langle f\left\|\sum_{j}^{A} t_{j}^{ \pm}\right\| i\right\rangle^{2}=T(T+1)-T_{z_{i}} T_{z_{f}},
$$

where $T$ is the nuclear isospin and $T_{z}=(N-Z) / 2$ is its third component. The $B_{i f}\left(F^{+}\right)$strength that we are concerned with here reduces to $B\left(F^{+}\right)=(Z-N)=2$ for the $\left(T, T_{z}\right)=$ $(1,-1)$ isotopes in the decay $(Z, N) \rightarrow(Z-1, N+1)$ with $Z=N+2$. For these transitions the excitation energy of the 

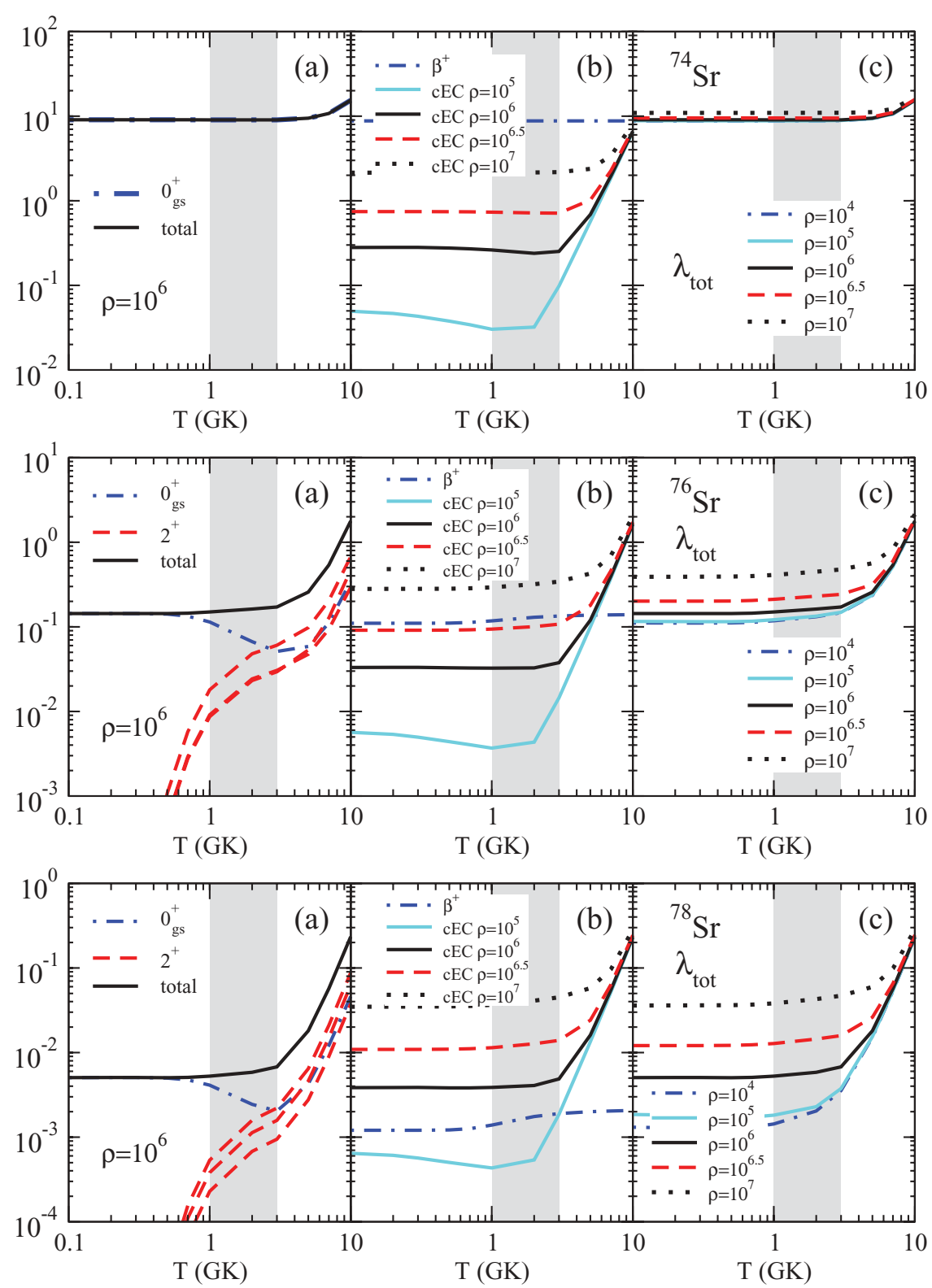

IAS in the daughter nucleus is given by $[8,10]$

$$
E_{\mathrm{IAS}}=(M E)_{i}-(M E)_{f}+0.7824-\Delta E_{C} \mathrm{MeV},
$$

where $M E$ is the atomic mass excess. The Coulomb displacement energy $\Delta E_{C}$ between pairs of isobaric analog levels is given by

$$
\Delta E_{C}=1.4144 \bar{Z} / A^{1 / 3}-0.9127 \mathrm{MeV},
$$

where $\bar{Z}=\left(Z_{i}+Z_{f}\right) / 2$. This expression was obtained in Ref. [41] from a fitting to data corresponding to levels with isospin $T=1$. In any case, Fermi transitions are only important for the $\beta^{+}$decay of neutron-deficient light nuclei with $Z>N\left(T_{z}<0\right)$, where the IAS can be reached energetically. Thus, although they have been considered in the calculations of the terrestrial half-lives, only the dominant GT transitions are included in the stellar decay rates.
FIG. 10. (Color online) Same as in Fig. 5 but for ${ }^{74,76,78} \mathrm{Sr}$ isotopes.

\section{B. Phase-space factors}

The phase-space factor contains two components, electron capture (EC) and $\beta^{+}$decay:

$$
\Phi_{i f}=\Phi_{i f}^{\mathrm{EC}}+\Phi_{i f}^{\beta^{+}}
$$

In the case of $\beta^{+} / \mathrm{EC}$ decay in the laboratory, EC arises from orbital electrons in the atom, and the phase-space factor is given by [42]

$$
\Phi^{\mathrm{OEC}}=\frac{\pi}{2} \sum_{x} q_{x}^{2} g_{x}^{2} B_{x},
$$

where $x$ denotes the atomic subshell from which the electron is captured, $q$ is the neutrino energy, $g$ is the radial component of the bound-state electron wave function at the nucleus, and $B$ stands for other exchange and overlap corrections [42]. 

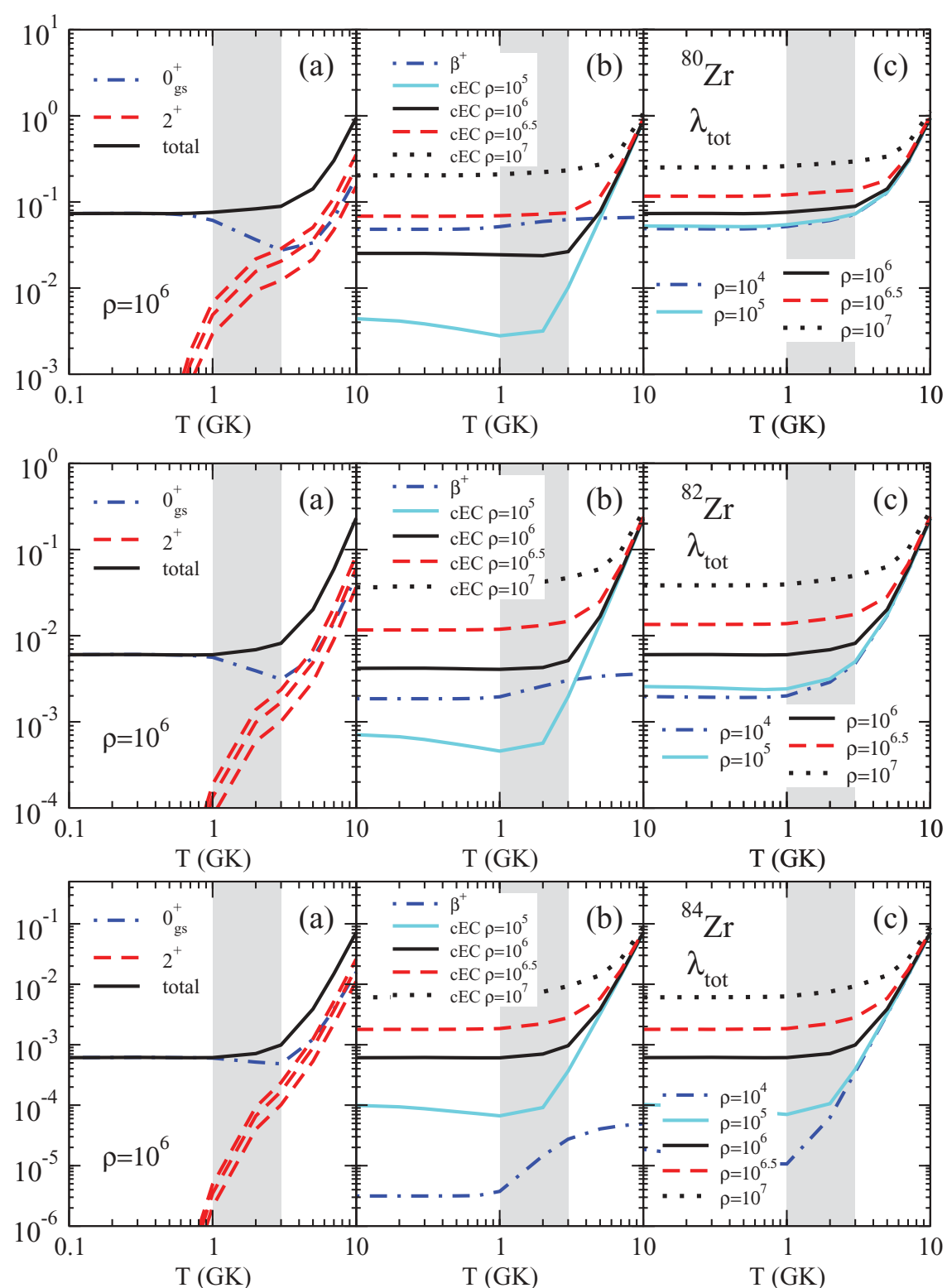

FIG. 11. (Color online) Same as in Fig. 5 but for ${ }^{80,82,84} \mathrm{Zr}$ isotopes.
In $r p$ process stellar scenarios, the phase-space factor for $\mathrm{CEC}$ is given by

$$
\begin{aligned}
\Phi_{i f}^{\mathrm{CEC}}= & \int_{\omega_{\ell}}^{\infty} \omega p\left(Q_{i f}+\omega\right)^{2} F(Z, \omega) \\
& \times S_{e^{-}}(\omega)\left[1-S_{v}\left(Q_{i f}+\omega\right)\right] d \omega .
\end{aligned}
$$

The phase-space factor for positron emission $\beta^{+}$process is given by

$$
\begin{aligned}
\Phi_{i f}^{\beta^{+}}= & \int_{1}^{Q_{i f}} \omega p\left(Q_{i f}-\omega\right)^{2} F(-Z+1, \omega) \\
& \times\left[1-S_{e^{+}}(\omega)\right]\left[1-S_{v}\left(Q_{i f}-\omega\right)\right] d \omega .
\end{aligned}
$$

In these expressions $\omega$ is the total energy of the positron in $m_{e} c^{2}$ units, $p=\sqrt{\omega^{2}-1}$ is the momentum in $m_{e} c$ units, and $Q_{i f}$ is the total energy available in $m_{e} c^{2}$ units:

$$
Q_{i f}=\frac{1}{m_{e} c^{2}}\left(M_{p}-M_{d}+E_{i}-E_{f}\right),
$$

which is is written in terms of the nuclear masses of parent $\left(M_{p}\right)$ and daughter $\left(M_{d}\right)$ nuclei and their excitation energies $E_{i}$ and $E_{f}$, respectively. $F(Z, \omega)$ is the Fermi function [42] that takes into account the distortion of the $\beta$-particle wave function due to the Coulomb interaction.

$$
F(Z, \omega)=2(1+\gamma)(2 p R)^{-2(1-\gamma)} e^{\pi y} \frac{|\Gamma(\gamma+i y)|^{2}}{[\Gamma(2 \gamma+1)]^{2}},
$$

where $\gamma=\sqrt{1-(\alpha Z)^{2}}, y=\alpha Z \omega / p, \alpha$ is the fine structure constant, and $R$ is the nuclear radius. The lower integration limit in the CEC expression is given by $\omega_{\ell}=1$ if $Q_{\text {if }}>-1$ or $\omega_{\ell}=\left|Q_{i f}\right|$ if $Q_{i f}<-1$.

$S_{e^{-}}, S_{e^{+}}$, and $S_{v}$ are the electron, positron, and neutrino distribution functions, respectively. Their presence inhibits or enhances the phase space available. In $r p$ scenarios the commonly accepted assumptions [5] state that $S_{v}=0$ since neutrinos and antineutrinos can escape freely from the interior of the star and then they do not block the emission of these 

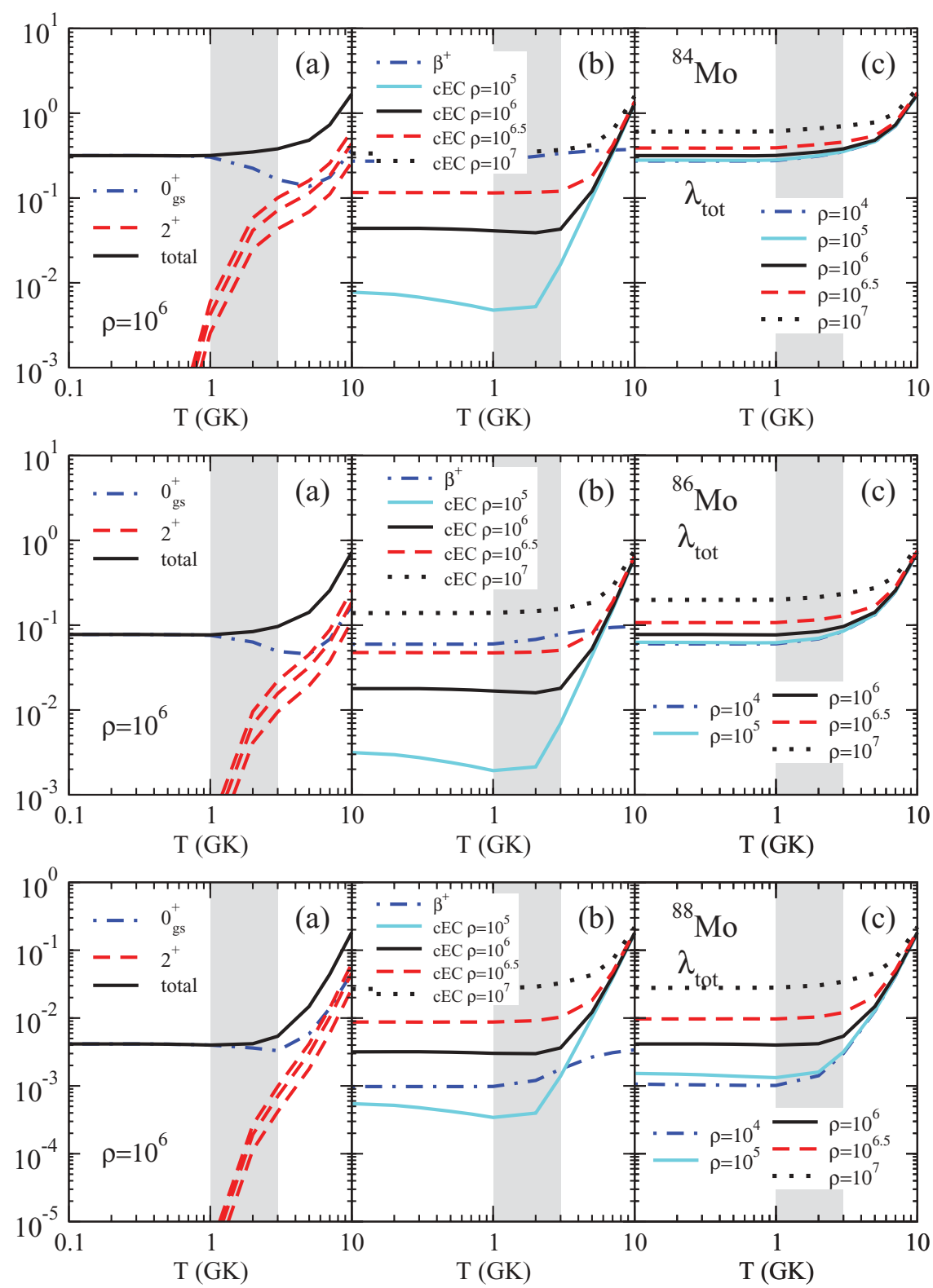

FIG. 12. (Color online) Same as in Fig. 5 but for ${ }^{84,86,88}$ Mo isotopes. particles in the capture or decay processes. Positron distributions become important only at higher $T(k T>1 \mathrm{MeV})$ when positrons appear via pair creation, but at the temperatures considered here we take $S_{e^{+}}=0$. The electron distribution is described as a Fermi-Dirac distribution:

$$
S_{e}=\frac{1}{\exp \left[\left(\omega-\mu_{e}\right) /(k T)\right]+1},
$$

assuming that nuclei at these temperatures are fully ionized and electrons are not bound to nuclei. The chemical potential $\mu_{e}$ is determined from the expression

$$
\rho Y_{e}=\frac{1}{\pi^{2} N_{A}}\left(\frac{m_{e} c}{\hbar}\right)^{3} \int_{0}^{\infty}\left(S_{e}-S_{e^{+}}\right) p d p
$$

in $\mathrm{mol} / \mathrm{cm}^{3} . \rho$ is the baryon density $\left(\mathrm{g} / \mathrm{cm}^{3}\right), Y_{e}$ is the electronto-baryon ratio $(\mathrm{mol} / \mathrm{g})$, and $N_{A}$ is Avogadro's number $\left(\mathrm{mol}^{-1}\right)$.
Under the assumption $S_{e^{+}}=S_{v}=0$, the phase-space factors for $\beta^{+}$decay in Eq. (20) are independent of the density and temperature. The only dependence of the $\beta^{+}$decay rates on $T$ arises from the thermal population of excited parent states. On the other hand, the phase-space factor for CEC in Eq. (19) is a function of both $\rho Y_{e}$ and $T$, through the electron distribution $S_{e^{-}}$.

The phase-space factors increase with $Q_{i f}$, and thus, the decay rates are more sensitive to the strength $B_{i f}$ located at low excitation energies of the daughter nucleus. It is also interesting to note the relative importance of both $\beta^{+}$decay and electroncapture phase-space factors (see Fig. 3 in Ref. [17]). In general, the former dominates at sufficiently high $Q_{i f}$ (low excitation energies in the daughter nucleus), while the latter is always dominant at sufficiently low $Q_{i f}$ (high excitation energies in the daughter nucleus).

The $\beta$-decay half-life in the laboratory is obtained by summing all the allowed transition strengths to states in the 

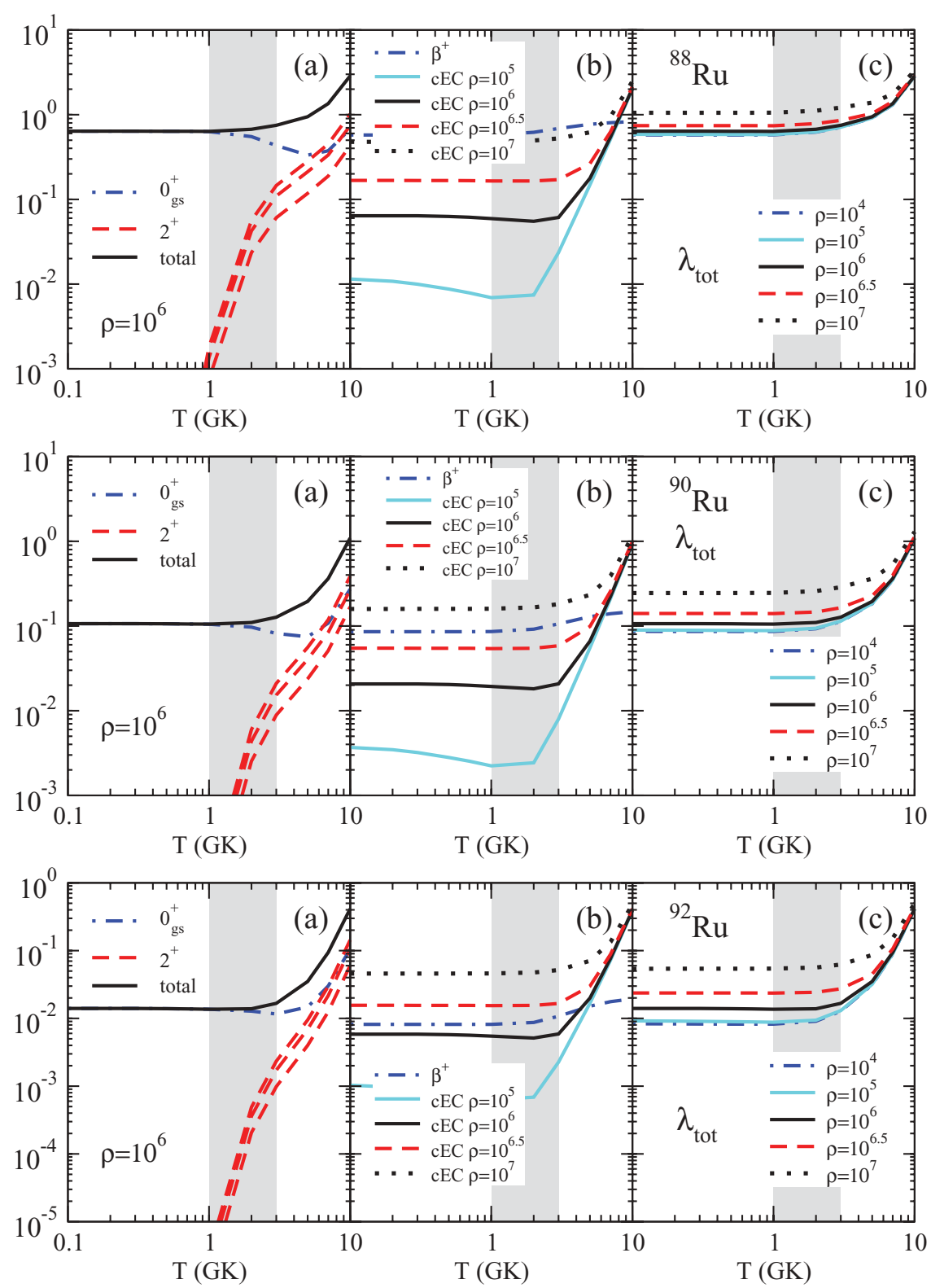

FIG. 13. (Color online) Same as in Fig. 5 but for ${ }^{88,90,92} \mathrm{Ru}$ isotopes. daughter nucleus with excitation energies lying below the corresponding $Q_{\mathrm{EC}}$ energy and weighted with the phase-space factors,

$$
T_{1 / 2}^{-1}=\frac{\lambda}{\ln 2}=\frac{1}{D} \sum_{0<E_{f}<Q_{\mathrm{EC}}}\left[B_{i f}(\mathrm{GT})+B_{i f}(\mathrm{~F})\right] \Phi_{i f}^{\beta^{+} / \mathrm{OEC}}
$$

where the $Q_{\mathrm{EC}}$ energy is given by

$$
Q_{\mathrm{EC}}=M_{p}-M_{d}+m_{e}=Q_{\beta^{+}}+2 m_{e} .
$$

\section{RESULTS FOR WEAK DECAY RATES}

In this section we first present the results for the potential energy curves. Then, we show the results for the decay properties, GT strength distributions, and $\beta$-decay half-lives under terrestrial conditions, comparing them with the available experimental information. Finally, we present the results for the stellar weak decay rates under the density and temperature conditions implied in the $r p$ process.

\section{A. Potential energy curves}

In Fig. 1 we can see the potential energy curves for the even-even $\mathrm{Ni}, \mathrm{Zn}, \mathrm{Ge}, \mathrm{Se}, \mathrm{Kr}, \mathrm{Sr}, \mathrm{Zr}, \mathrm{Mo}, \mathrm{Ru}, \mathrm{Pd}, \mathrm{Cd}$, and Sn nuclei in the vicinity of the $N=Z$ isotopes considered in this work. We show the energies relative to that of the ground state plotted as a function of the quadrupole deformation $\beta$ in Eq. (5). They are obtained from constrained HF + BCS calculations with the Skyrme force SLy4 [32].

The nuclei studied here cover a whole proton shell ranging from magic number $Z=28$ ( $\mathrm{Ni}$ isotopes) up to magic number $Z=50$ ( $\mathrm{Sn}$ isotopes). The isotopes considered are the predicted WP nuclei, which in most cases correspond to $N=Z$, and their neighbor isotopes. 

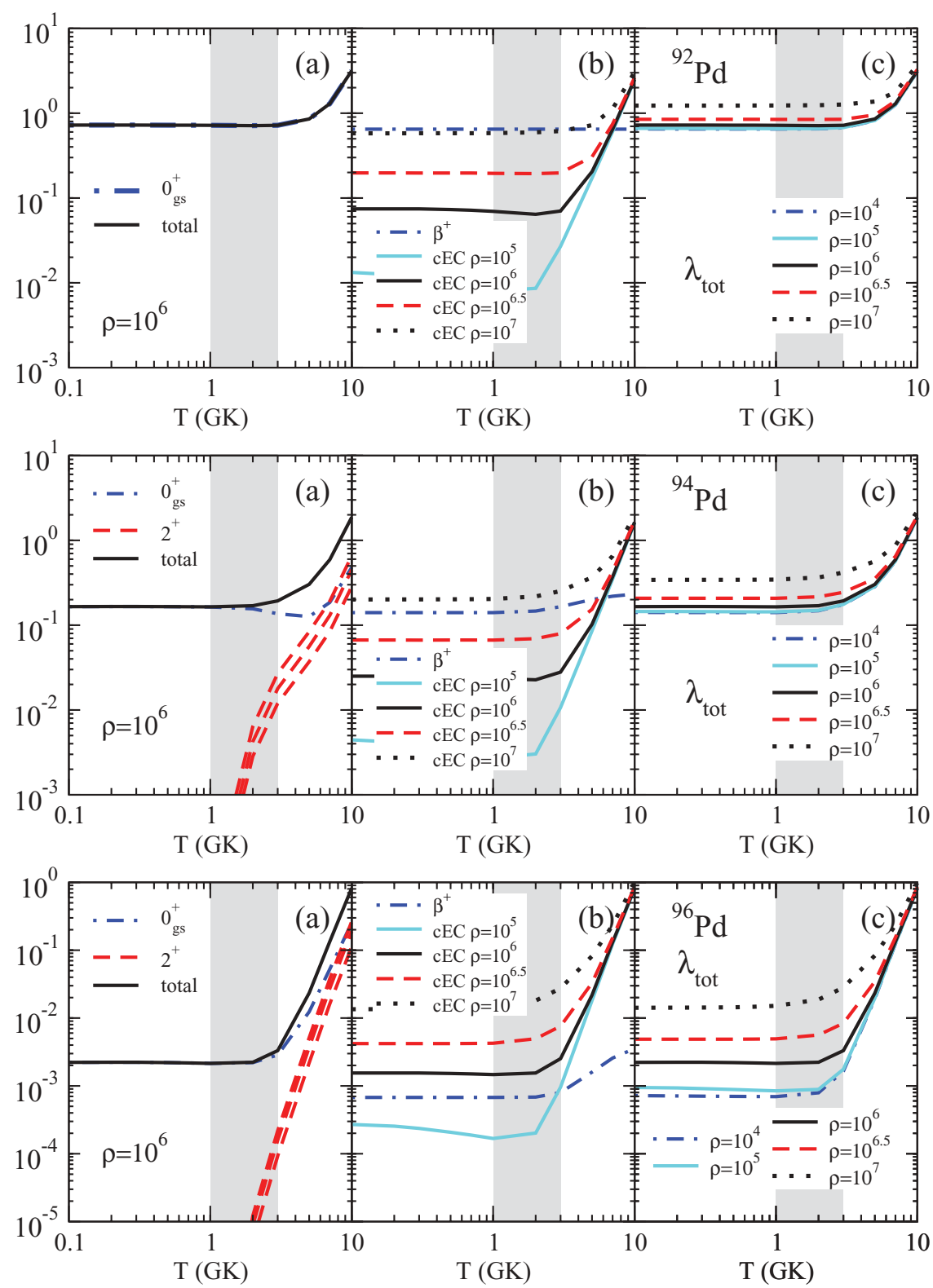

FIG. 14. (Color online) Same as in Fig. 5 but for ${ }^{92,94,96} \mathrm{Pd}$ isotopes.
Then, it is expected that the lighter and heavier nuclei close to $Z=28$ and $Z=50$, respectively, have a tendency to be spherical. The spherical shapes in these isotopes show sharply peaked profiles that become shallow minima as one moves away from $Z=28$ or $Z=50$, and finally, deformed shapes are developed as one approaches midshell nuclei. The profiles of the latter exhibit a rich structure giving ise to shape coexistence when various minima at close energies are located at different deformations.

It is also worth mentioning the correlations observed between mirror nuclei interchanging the number of neutrons and protons. Thus, we see a remarkable similarity between the profiles of ${ }^{66} \mathrm{Ge}(Z=32, N=34)$ and ${ }^{66} \mathrm{Se}(Z=$ $34, N=32)$, between ${ }^{70} \mathrm{Se}(Z=34, N=36)$ and ${ }^{70} \mathrm{Kr}(Z=$ $36, N=34)$, and between ${ }^{74} \mathrm{Kr}(Z=36, N=38)$ and ${ }^{74} \mathrm{Sr}$ $(Z=38, N=36)$.

These results are in qualitative agreement with similar ones obtained in this mass region from different theoretical approaches. Just to give some examples, shape transition and shape coexistence were discussed in $A \sim 80$ nuclei within a configuration-dependent shell-correction approach based on a deformed Woods-Saxon potential [43]. Relativistic mean-field calculations in this mass region have also been reported in Ref. [44]. Nonrelativistic calculations are also available from both Skyrme [45-47] and Gogny [48] forces, as well as from the complex VAMPIR approach [49].

Experimental evidence of shape coexistence in this mass region has become available in recent years [50-65], and by now this is a well-established characteristic feature in the neutron-deficient $A=70-80$ mass region.

\section{B. Laboratory Gamow-Teller strength and half-lives}

While the half-lives give only limited information about the decay (different strength distributions may lead to the 

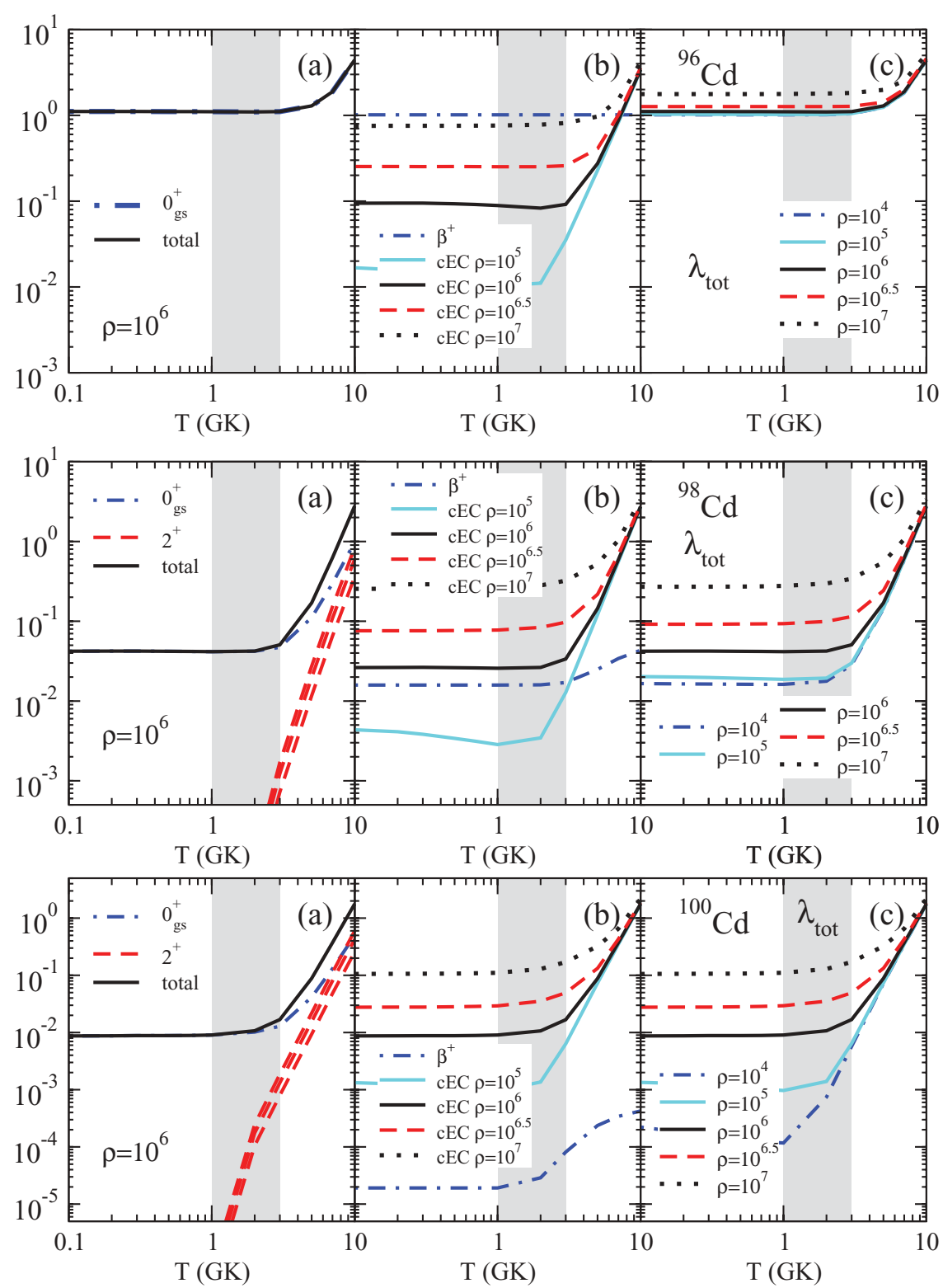

FIG. 15. (Color online) Same as in Fig. 5 but for ${ }^{96,98,100} \mathrm{Cd}$ isotopes. same half-life), the strength distribution contains all the information. It is of great interest to study the decay rates under stellar $r p$ conditions using a nuclear structure model that reproduces the strength distributions and half-lives under terrestrial conditions.

In Figs. 2 and 3, we show the results obtained for the energy distributions of the GT strength corresponding to the equilibrium shapes for which we obtained minima in the potential energy curves in Fig. 1. The GT strength is plotted versus the excitation energy of the daughter nucleus $E_{e x}=$ $E_{f}(\mathrm{MeV})$.

Figure 2 (Fig. 3) contains the results for the isotopes $\mathrm{Ni}$, $\mathrm{Zn}, \mathrm{Ge}, \mathrm{Se}, \mathrm{Kr}$, and $\mathrm{Sr}$ (Zr, Mo, Ru, Pd, Cd, and Sn). We show the energy distributions of the individual GT strengths in the case of the ground-state shapes. We also show the continuous distributions for both ground-state and possible shape isomers, obtained by folding the strength with $1 \mathrm{MeV}$ width BreitWigner functions. The vertical arrows show the $Q_{\mathrm{EC}}$ energy and the proton separation energy in the daughter nucleus, both taken from experiment [34].

It is worth noting that, in general, both deformations produce quite similar GT strength distributions on a global scale. The main exceptions correspond to the comparison between spherical and deformed shapes, where clear differences can be observed. In any case, the small differences among the various shapes at the low-energy tails (below the $Q_{\mathrm{EC}}$ ) of the GT strength distributions lead to sizable effects in the $\beta$-decay half-lives. These differences can be better seen because of the logarithmic scale.

Experimental information on GT strength distributions are mainly available for ${ }^{72} \mathrm{Kr}$ [66], ${ }^{74} \mathrm{Kr}$ [67], ${ }^{76} \mathrm{Sr}$ [68], and ${ }^{102,104} \mathrm{Sn}$ [69] isotopes, where $\beta^{+}$-decay experiments have been performed with total absorption spectroscopy techniques, allowing the extraction of the GT strength in practically the whole $Q$-energy window. In Ref. [18] a comparison between similar calculations to those in this 

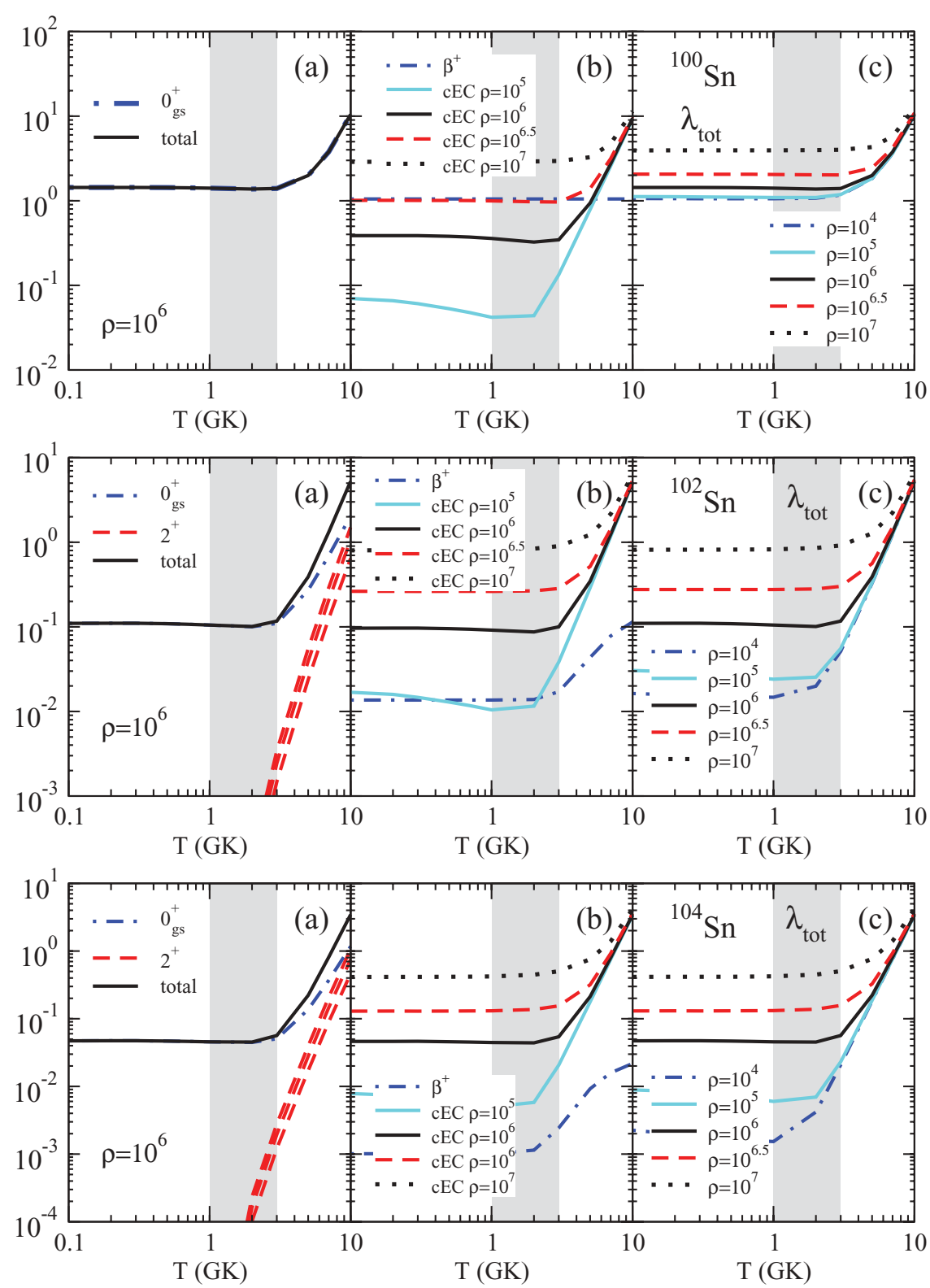

FIG. 16. (Color online) Same as in Fig. 5 but for ${ }^{100,102,104} \mathrm{Sn}$ isotopes. work and the experimental data for $\mathrm{Kr}$ and $\mathrm{Sr}$ isotopes was carried out. In general, good agreement with experiments was found, and this was one of the reasons to extrapolate this type of calculation to stellar environments and to other WP nuclei.

Measurements of the decay properties (mainly half-lives) of nuclei in this mass region have been reported in recent years [69-80]. The calculation of the half-lives in Eq. (25) involves knowledge of the GT strength distribution and of the $Q_{\text {EC }}$ values. In this work, experimental values for $Q_{\mathrm{EC}}$ are used. They are taken from Ref. [34] or from the Jyväskylä mass database [79,81], when available. In Fig. 4 the measured half-lives are compared to the QRPA results obtained from the equilibrium deformations of the various isotopes. In general, good agreement for the $N=Z \mathrm{WP}$ is obtained. Also, for the more stable $N=Z+2$, the agreement is very reasonable, except for the heavier $\mathrm{Cd}$ and $\mathrm{Sn}$ isotopes, where the half-lives are overestimated. The half-lives of the more exotic isotopes are fairly well described by QRPA.

\section{Stellar weak decay rates}

Figures 5-16 show the decay rates as a function of the temperature $T$. In Figs. 5(a)-16(a) one can see the decomposition of the total rates into their contributions from the decay of the the ground state $0_{\mathrm{gs}}^{+} \rightarrow 1^{+}$and from the decay of the excited state $2^{+} \rightarrow 1^{+}, 2^{+}, 3^{+}$in the parent nucleus. Figures 5(b)-16(b) show the decomposition of the rates into their $\beta^{+}$and CEC components evaluated at various densities $\left(\rho Y_{e}\right)$. Figures 5(c)-16(c) the total rates for various densities. The gray area is the relevant range $T=1-3 \mathrm{GK}$ for the $r p$ process. Each figure contains the results for three isotopes. The results corresponding to the more exotic ones are displayed in the top plots, whereas the results corresponding to the 
more stable isotopes appear in the bottom plots. In the middle plots we give the intermediate isotopes, which, in most cases, correspond to the WP nuclei.

The results decomposed into their contributions from various parent states [Figs. 5(a)-16(a)] show that the decay from the ground state is always dominant at the temperatures within the gray area of interest. The contributions of the decays from excited states increase with $T$, as they become more and more thermally populated, but in general, they do not represent significant contributions to the total rates and can be neglected in most cases. Nevertheless, there are a few cases where these contributions should not be ignored, which correspond to those cases where the excitation energy of the $2^{+}$excited state is very low. This is the case of the middle-shell nuclei $\mathrm{Kr}, \mathrm{Sr}, \mathrm{Zr}$, and $\mathrm{Mo}$, where the contributions of the low-lying excited states compete with those of the ground state already at temperatures in the range of $r p$ process. The effect on the rates of the decay from excited $\mathrm{O}_{2}^{+}$states was also considered in Ref. [17] in the case of $\mathrm{Kr}$ and $\mathrm{Sr}$ isotopes. It was concluded that, in general, their relative impact is again very small in the total rates at these temperatures.

Concerning the competition between $\beta^{+}$and CEC rates [Figs. 5(b)-16(b)], one should distinguish between different isotopes. Thus, the more exotic isotopes appearing in the top plots of the figures show a clear dominance of the $\beta^{+}$ rates over the CEC ones, which can be neglected except at very high densities beyond $r p$-process conditions. On the other hand, the opposite is true with respect to the more stable isotopes in the bottom plots, where the $\beta^{+}$rates are completely negligible. The origin of these features can be understood from the behavior of the phase-space factors as a function of the available energy $Q_{i f}$. As mentioned in Sec. II B and discussed in Ref. [17], more exotic nuclei with larger $Q_{i f}$ values favor $\beta^{+}$because of the larger phase-space factors, while the opposite is true for more stable nuclei with smaller $Q_{i f}$ values.

The interesting cases occur in the middle panels, which correspond in most cases to the $N=Z$ WP nuclei.In these plots, there is a competition between $\beta^{+}$and CEC rates that depends on the nucleus, on the temperature, and on the density $\rho Y_{e}$. One can see that for large enough densities, CEC becomes dominant at any $T$. For low densities, $\beta^{+}$rates dominate at low
$T$, while CEC dominates at higher $T$, but in general, there is a competition that must be analyzed case by case.

Finally, the total rates in Figs. 5(c)-16(c) are a consequence of the competition between $\beta^{+}$and CEC rates. Since the $\beta^{+}$ decay rate is independent of the density and depends on $T$ only through the contributions from excited parent states, the total rates are practically constant for the more exotic isotopes in the top plots, except modulated by the small contribution from CEC. In the central isotopes the rates are the result of the competition shown in Figs. 5(b)-16(b), and finally, in the heavier isotopes (bottom plots) we can see that the total rates are practically due to CEC with little contribution from $\beta^{+}$. Tables containing $\beta^{+}$, CEC, and total decay rates for all the isotopes considered in this work are available in Ref. [82].

\section{SUMMARY AND CONCLUSIONS}

In summary, the weak decay rates of waiting-point and neighbor nuclei from $\mathrm{Ni}$ up to $\mathrm{Sn}$ have been investigated at temperatures and densities where the $r p$ process takes place. The nuclear structure has been described within a microscopic QRPA approach based on a self-consistent Skyrme-HartreeFock-BCS mean field that includes deformation. This approach reproduces both the experimental half-lives and the more demanding GT strength distributions measured under terrestrial conditions in this mass region.

The relevant ingredients to describe the rates have been analyzed. We have studied the contributions to the decay rates coming from excited states in the parent nucleus, which are populated as $T$ increases. It is found that they start to play a role above $T=1-2 \mathrm{GK}$ and that for isotopes with lowlying excited states, their contributions can be comparable to those of the ground states. Concerning the contributions from the continuum electron-capture rates, it is found that they are enhanced with $T$ and $\rho$. They are already comparable to the $\beta^{+}$decay rates at $r p$ conditions for the WP nuclei. For more exotic isotopes the rates are dominated by $\beta^{+}$decay, while for more stable isotopes they are dominated by CEC.

\section{ACKNOWLEDGMENTS}

This work was supported by the Ministerio de Ciencia e Innovación (Spain) under Contract No. FIS2008-01301.
[1] K. Langanke and G. Martínez-Pinedo, Rev. Mod. Phys. 75, 819 (2003).

[2] A. Aprahamian, K. Langanke, and M. Wiescher, Prog. Part. Nucl. Phys. 54, 535 (2005).

[3] R. K. Wallace and S. E. Woosley, Astrophys. J. Suppl. Ser. 45, 389 (1981)

[4] F.-K. Thielemann et al., Nucl. Phys. A 570, 329c (1994).

[5] H. Schatz et al., Phys. Rep. 294, 167 (1998).

[6] S. E. Woosley et al., Astrophys. J. Suppl. Ser. 151, 75 (2004).

[7] L. Van Wormer et al., Astrophys. J. 432, 326 (1994).

[8] J. Pruet and G. M. Fuller, Astrophys. J. Suppl. Ser. 149, 189 (2003).

[9] H. Schatz et al., Phys. Rev. Lett. 86, 3471 (2001).
[10] G. M. Fuller, W. A. Fowler, and M. J. Newman, Astrophys. J. Suppl. Ser. 42, 447 (1980); Astrophys. J. 252, 715 (1982); Astrophys. J. Suppl. Ser. 48, 279 (1982); Astrophys. J. 293, 1 (1985)

[11] S. Wanajo, Astrophys. J. 647, 1323 (2006).

[12] C. Fröhlich et al., Phys. Rev. Lett. 96, 142502 (2006).

[13] K. Langanke and G. Martínez-Pinedo, Nucl. Phys. A 673, 481 (2000).

[14] K. Langanke and G. Martínez-Pinedo, At. Data Nucl. Data Tables 79, 1 (2001).

[15] J.-U. Nabi and H. V. Klapdor-Kleingrothaus, At. Data Nucl. Data Tables 71, 149 (1999); 88, 237 (2004).

[16] N. Paar, G. Colò, E. Khan, and D. Vretenar, Phys. Rev. C 80, 055801 (2009). 
[17] P. Sarriguren, Phys. Lett. B 680, 438 (2009).

[18] P. Sarriguren, Phys. Rev. C 79, 044315 (2009).

[19] Evaluated Nuclear Structure Data File (ENSDF), [http://www. nndc.bnl.gov/ensdf/].

[20] J. Krumlinde and P. Möller, Nucl. Phys. A 417, 419 (1984); P. Möller and J. Randrup, ibid. 514, 1 (1990).

[21] F. Frisk, I. Hamamoto, and X. Z. Zhang, Phys. Rev. C 52, 2468 (1995).

[22] P. Möller, J. R. Nix, and K. -L. Kratz, At. Data Nucl. Data Tables 66, 131 (1997).

[23] M. Hirsch, A. Staudt, K. Muto, and H. V. KlapdorKleingrothaus, Nucl. Phys. A 535, 62 (1991); K. Muto, E. Bender, T. Oda, and H. V. Klapdor-Kleingrothaus, Z. Phys. A Hadrons Nucl. 341, 407 (1992).

[24] H. Homma, E. Bender, M. Hirsch, K. Muto, H. V. KlapdorKleingrothaus, and T. Oda, Phys. Rev. C 54, 2972 (1996).

[25] I. N. Borzov, Nucl. Phys. A 777, 645 (2006).

[26] N. Paar, T. Nikšić, D. Vretenar, and P. Ring, Phys. Rev. C 69, 054303 (2004).

[27] S. Fracasso and G. Colò, Phys. Rev. C 76, 044307 (2007).

[28] A. Petrovici, K. W. Schmid, O. Radu, and A. Faessler, Nucl. Phys. A 799, 94 (2008); Phys. Rev. C 78, 044315 (2008); A. Petrovici, K. W. Schmid, O. Andrei, and A. Faessler, ibid. 80, 044319 (2009).

[29] P. Sarriguren, E. Moya de Guerra, A. Escuderos, and A. C. Carrizo, Nucl. Phys. A 635, 55 (1998).

[30] P. Sarriguren, E. Moya de Guerra, and A. Escuderos, Nucl. Phys. A 691, 631 (2001).

[31] P. Sarriguren, E. Moya de Guerra, and A. Escuderos, Phys. Rev. C 64, 064306 (2001)

[32] E. Chabanat et al., Nucl. Phys. A 635, 231 (1998).

[33] D. Vautherin, Phys. Rev. C 7, 296 (1973).

[34] G. Audi, O. Bersillon, J. Blachot, and A. H. Wapstra, Nucl. Phys. A 729, 3 (2003).

[35] H. Flocard, P. Quentin, A. K. Kerman, and D. Vautherin, Nucl. Phys. A 203, 433 (1973).

[36] P. Sarriguren, E. Moya de Guerra, and A. Escuderos, Nucl. Phys. A 658, 13 (1999).

[37] P. Sarriguren, O. Moreno, R. Álvarez-Rodríguez, and E. Moya de Guerra, Phys. Rev. C 72, 054317 (2005).

[38] P. Sarriguren, R. Álvarez-Rodríguez, and E. Moya de Guerra, Eur. Phys. J. A 24, 193 (2005).

[39] P. Sarriguren and J. Pereira, Phys. Rev. C 81, 064314 (2010).

[40] A. Bohr and B. Mottelson, Nuclear Structure, (Benjamin, New York, 1975).

[41] M. S. Antony, A. Pape, and J. Britz, At. Data Nucl. Data Tables 66, 1 (1997).

[42] N. B. Gove and M. J. Martin, Nucl. Data Tables 10, 205 (1971).

[43] W. Nazarewicz, J. Dudek, R. Bengtsson, T. Bengtsson, and I. Ragnarsson, Nucl. Phys. A 435, 397 (1985).

[44] G. A. Lalazissis and M. M. Sharma, Nucl. Phys. A 586, 201 (1995); G. A. Lalazissis, S. Raman, and P. Ring, At. Data Nucl. Data Tables 71, 1 (1999).
[45] P. Bonche, H. Flocard, P.-H. Heenen, S. J. Krieger, and M. S. Weiss, Nucl. Phys. A 443, 39 (1985).

[46] M. Bender, P. Bonche, and P.-H. Heenen, Phys. Rev. C 74, 024312 (2006).

[47] M. Yamagami, K. Matsuyanagi, and M. Matsuo, Nucl. Phys. A 693, 579 (2001).

[48] S. Hilaire and M. Girod, Eur. Phys. J. A 33, 237 (2007); M. Girod, J.-P. Delaroche, A. Görgen, and A. Obertelli, Phys. Lett. B 676, 39 (2009).

[49] A. Petrovici, K. W. Schmid, and A. Faessler, Nucl. Phys. A 605, 290 (1996); 665, 333 (2000).

[50] J. L. Wood, E. F. Aganjar, C. de Coster, and K. Heyde, Nucl. Phys. A 651, 323 (1999).

[51] R. B. Piercey et al., Phys. Rev. Lett. 47, 1514 (1981).

[52] C. Chandler et al., Phys. Rev. C 56, R2924 (1997).

[53] F. Becker et al., Eur. Phys. J. A 4, 103 (1999).

[54] S. M. Fischer, D. P. Balamuth, P. A. Hausladen, C. J. Lister, M. P. Carpenter, D. Seweryniak, and J. Schwartz, Phys. Rev. Lett. 84, 4064 (2000).

[55] E. Bouchez et al., Phys. Rev. Lett. 90, 082502 (2003).

[56] A. Gade et al., Phys. Rev. Lett. 95, 022502 (2005).

[57] A. Görgen et al., Eur. Phys. J. A 26, 153 (2005).

[58] E. Clément et al., Phys. Rev. C 75, 054313 (2007).

[59] P. J. Davies et al., Phys. Rev. C 75, 011302(R) (2007).

[60] C. Andreoiu et al., Phys. Rev. C 75, 041301(R) (2007).

[61] B. S. Nara Singh et al., Phys. Rev. C 75, 061301(R) (2007).

[62] A. M. Hurst et al., Phys. Rev. Lett. 98, 072501 (2007).

[63] A. Görgen et al., Eur. Phys. J. Spec. Top. 150, 117 (2007).

[64] J. Ljungvall et al., Phys. Rev. Lett. 100, 102502 (2008).

[65] A. Obertelli et al., Phys. Rev. C 80, 031304(R) (2009).

[66] I. Piqueras et al., Eur. Phys. J. A 16, 313 (2003).

[67] E. Poirier et al., Phys. Rev. C 69, 034307 (2004).

[68] E. Nácher et al., Phys. Rev. Lett. 92, 232501 (2004).

[69] M. Karny et al., Eur. Phys. J. A 27, 129 (2006).

[70] M. Oinonen et al., Phys. Rev. C 61, 035801 (2000).

[71] P. Kienle et al., Prog. Part. Nucl. Phys. 46, 73 (2001).

[72] T. Faestermann et al., Eur. Phys. J. A 15, 185 (2002).

[73] A. Wöhr et al., Nucl. Phys. A 742, 349 (2004).

[74] A. Kankainen et al., Eur. Phys. J. A 29, 271 (2006).

[75] O. Kavatsyuk et al., Eur. Phys. J. A 31, 319 (2007).

[76] C. Dossat et al., Nucl. Phys. A 792, 18 (2007).

[77] D. Bazin et al., Phys. Rev. Lett. 101, 252501 (2008).

[78] J. B. Stoker et al., Phys. Rev. C 79, 015803 (2009).

[79] C. Weber et al., Phys. Rev. C 78, 054310 (2008).

[80] V.-V. Elomaa et al., Eur. Phys. J. A 40, 1 (2009); Phys. Rev. Lett. 102, 252501 (2009).

[81] [http://research.jyu.fi/igisol/JYFLTRAP_masses/].

[82] See supplemental material at [http://link.aps.org/supplemental/ 10.1103/PhysRevC.83.025801] for text files containing weak decay rates in a grid of densities $\left(\rho Y_{e}\right)$ and temperatures $(T)$ covering the $r p$-process conditions. 